\title{
Etat des lieux de la gestion des déchets dans le Grand Lomé
}

\author{
Essowè KONDOH ${ }^{1,2}$, Magnoudéwa Bassaï BODJONA ${ }^{1,2^{*}}$, Etsè AZIABLE ${ }^{1}$, \\ Sanonka TCHEGUENI ${ }^{1,2}$, Koffi Ani KILI ${ }^{1,2}$ et Gado TCHANGBEDJI ${ }^{1,2}$ \\ ${ }^{1}$ Laboratoire Gestion, Traitement et Valorisation des Déchets (GTVD), Faculté Des Sciences, Université de \\ Lomé ; BP 1515 Lomé, Togo. \\ ${ }^{2}$ Centre d'Excellence Régional Ville Durable en Afrique (CERVIDA) : DOUNEDON, Université de Lomé ; BP \\ 1515 Lomé, Togo. \\ *Auteur correspondant; E-mail: bodjonabenoit@yahoo.fr/bodjonabassai@gmail.com; Tél : (00228) 90125411
}

\section{RESUME}

La ville de Lomé et ses environs (Grand Lomé) à l'instar des autres villes africaines rencontrent des difficultés dans la gestion des déchets. L'objectif de ce travail est de faire le diagnostic de la gestion des déchets dans le Grand Lomé. Les enquêtes ont été menées sur deux types d'acteurs de la gestion des déchets, notamment les formels et informels, dans cinq zones en suivant le prolongement des cinq arrondissements que compte la commune de Lomé. Dans le Grand Lomé, on distingue trois systèmes de collecte des déchets notamment la collecte directe, la pré-collecte et la collecte des dépotoirs intermédiaires vers la décharge finale. Trois acteurs clés se partagent la tâche de la gestion des déchets: la collectivité, le secteur formel et le secteur informel. La production moyenne annuelle de déchets dans le Grand Lomé est estimée à 305340 tonnes dont 89428 sont collectés et enfouis soit un taux de collecte de 29,29\%. Une infime partie des déchets collectés (9000 tonnes soit 2,95\%) est valorisée en compost par les associations et/ou ONG. Une meilleure organisation de la gestion des déchets dans la ville de Lomé permettra de réduire la quantité de déchets à enfouir et d'assainir d'avantage la ville.

(C) 2019 International Formulae Group. All rights reserved.

Mots clés: Grand Lomé, déchets, gestion, collecte, dépotoirs, décharge finale.

\section{Inventory of waste management in Greater Lomé}

\begin{abstract}
The city of Lomé and its surroundings (Grand Lomé) like other African cities encounter difficulties in waste management. The objective of this work is to make waste management diagnosis in Grand Lomé. The surveys were conducted on two types of actors of waste management, including formal and informal, in five zones, following the prolongment of the five districts in the Lomé municipality. In Greater Lomé, there are three waste collection systems including direct collection, pre-collection and collection of intermediate dumps to the final discharge. Three key actors share the task of waste management: the collectivity, the formal sector and the informal sector. The average annual production of waste in Greater Lomé is estimated at 305340 tons of which 89428 are collected and buried, a collection rate of $29.29 \%$. A small part of the waste collected (9000 tonnes or $2.95 \%$ ) is valued as waste composted by the associations and / or NGOs.
\end{abstract}


A better organization of waste management in the city of Lomé will reduce the amount of waste to bury and sanitize the city.

(C) 2019 International Formulae Group. All rights reserved.

Keywords: Grand Lomé, wastes, management, collection, dumps, final discharge.

\section{INTRODUCTION}

La gestion des déchets dans les villes africaines constitue un enjeu majeur du point de vue technique et organisationnel (Koledzi et al., 2014). Avec une croissance rapide de la population entrainant une augmentation significative de la quantité de déchets, les structures mises en place par les municipalités peinent à assurer la gestion de ces déchets. Cette insuffisance est liée d'une part à la carence de ressource financière et d'autre part à une mauvaise organisation de la filière. Selon Moreno-Sánchez et Maldonado (2006), le taux de collecte dans les pays en développement se situe entre 50 et $80 \%$ des déchets générés, ce qui implique qu'une partie de la population, généralement celle à faible revenu, ne bénéficie pas de ce service. Les politiques d'assainissement pratiquement inexistantes ou n'étant pas encore la priorité de certaines villes, il n'est pas surprenant de constater la prolifération des dépotoirs sauvages dans certains quartiers. Malheureusement, ces dépotoirs sauvages reçoivent toutes les catégories de déchets voire les déchets biomédicaux de certaines cliniques et/ou cabinets médicaux. Ce fait pose beaucoup de problèmes sur le plan sanitaire et environnemental (Harpet, 2003 ; Aloueimine, 2006). L'absence d'un schéma directeur d'assainissement dans ces villes ne favorise pas une gestion efficiente et pérenne des déchets. Lomé, capitale du Togo n'est pas en marge de ces problèmes à savoir l'absence de centre de transit, mauvaise organisation des municipalités, l'absence de réglementation dans la profession de ramassage des solides urbains, etc. La gestion des déchets doit obéir à des normes afin de ne pas s'exposer à la pollution de l'environnement (Bodjona, 2012).

La collecte et la gestion des déchets est l'une des difficultés auxquelles sont confrontées les autorités municipales de Lomé. La gestion des déchets solides ménagers dans la ville de Lomé et ses environs, est un défi majeur qui doit être relevé au cas contraire, constitue une menace pour la santé publique, l'environnement et le développement socio-économique. En dépit des efforts déployés par les autorités municipales, ces déchets sont, soit brûlés par les habitants, soit transportés vers des dépotoirs non autorisés ou encore déposés dans les rues et espaces vides. D'autres acteurs de la gestion des déchets notamment les récupérateurs s'invitent dans la chaîne. L'implication des récupérateurs dans la chaine de la gestion des déchets est purement économique (Moreno-Sánchez et Maldonado, 2006). Ces récupérateurs jouent un rôle important dans la transformation des déchets en ressources d'une haute valeur marchande (Wilson et al., 2006). Le présent travail a pour objectif de faire l'état des lieux de la gestion des déchets dans le Grand Lomé (Cinq arrondissements de la commune de Lomé et ses environnants). Il est question de rechercher les maillons clés dans la chaine de gestion des déchets et d'évaluer la contribution des informels à partir d'une enquête de terrain.

\section{MATERIEL ET METHODES \\ Outils et enquête de terrain}

Les informations relatives aux différents acteurs intervenants dans la chaine de la gestion des déchets sont obtenues à travers un diagnostic sur le terrain en parcourant les services publics, privés et informels dans le Grand Lomé participants directement ou indirectement dans la précollecte, la collecte, mise en décharge et la valorisation des déchets.

L'observation et l'entretien sont les deux techniques utilisées pour la collecte des 
données. Les outils de terrain sont essentiellement les fiches d'enquêtes. Le questionnaire d'enquête a été élaboré et adressé aux acteurs informels identifiés selon le type d'activité, ceci pour connaitre leur réelle contribution à la gestion des déchets. Les enquêtes ont été menées sur un échantillon de 200 personnes de janvier à juillet 2017. La fiche d'enquête élaborée comporte deux types de questionnaire notamment des questionnaires sur l'identité des enquêtés, des questionnaires sur le travail qu'effectue les enquêtés. Les données ont été collectées à travers un entretien avec les informels de la gestion des déchets. Le nombre de personnes enquêtées dans chaque zone est fonction de la taille de la population dans les cinq zones. Ces zones constituent soit un arrondissement ou soit le prolongement d'un arrondissement que compte la commune de Lomé : Zone (1) 20 personnes, Zone (2) 70 personnes, Zone (3) 40 personnes, Zone (4) 20 personnes, Zone (5) 50 personnes (Figure 1).

\section{Traitement des données}

Le dépouillement des fiches d'enquêtes a été réalisé à l'aide du logiciel Microsoft Excel. Le traitement a débuté par la saisie des réponses aux questions et la mise en place d'une base de données regroupant l'ensemble des données collectées.

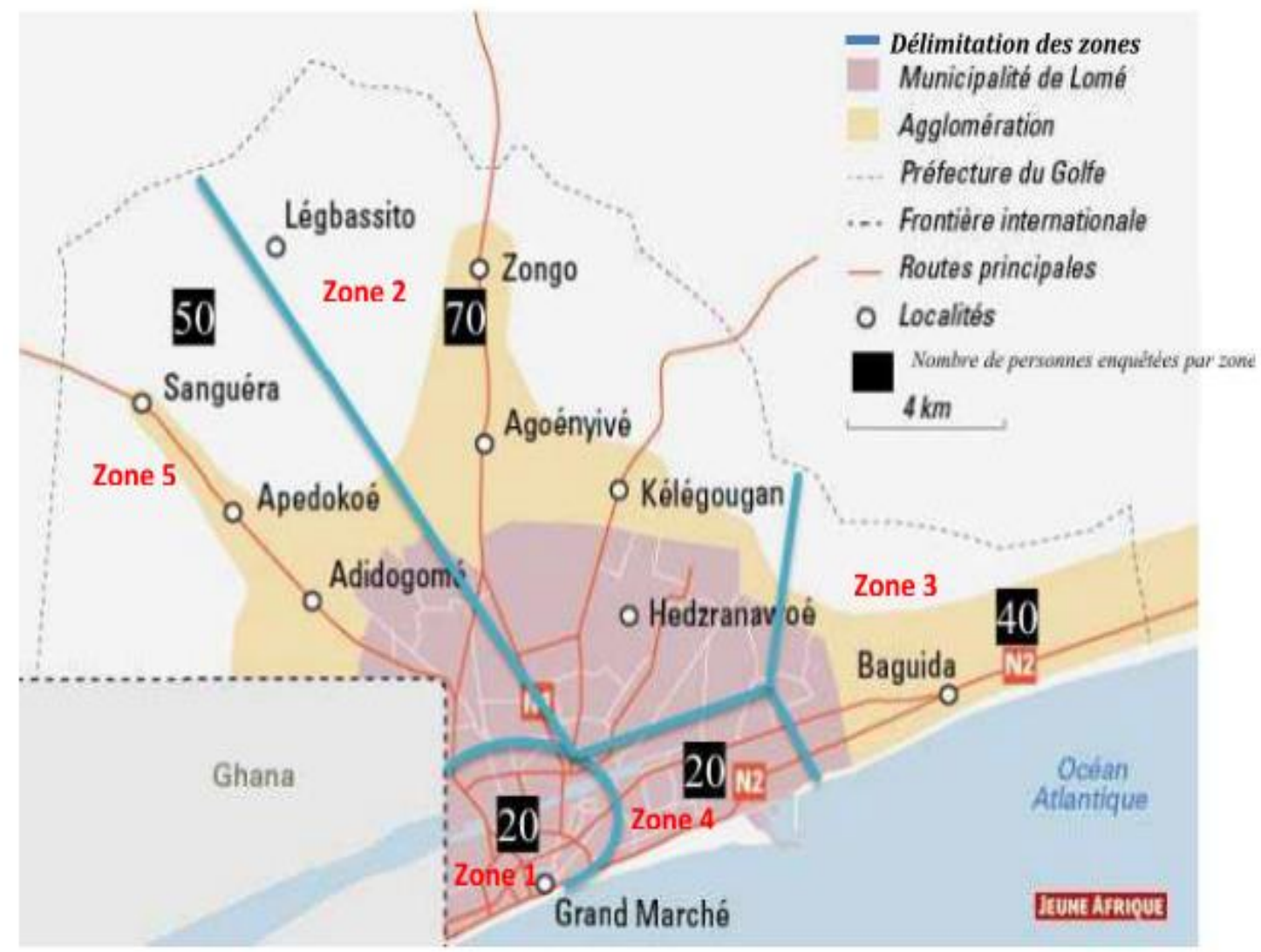

Figure 1: Carte du Grand Lomé indiquant les différentes enquêtées. 


\section{RESULTATS}

\section{Caractéristiques sociodémographiques des personnes enquêtées}

La figure 2 montre la répartition de tranche d'âge, le genre et le niveau d'instruction des personnes enquêtées. Les résultats du terrain révèlent que la majorité des personnes enquêtées a un âge compris entre 18 et 35 ans $(89 \%)$ et sont de sexe masculin (93\%) (Figure 2). En effet, les analphabètes sont environ de $17 \%$, niveau primaire $27 \%$, niveau secondaire $35 \%$, et niveau universitaire 1\% (Figure 2).

\section{Etat des lieux de la gestion des déchets dans le Grand Lomé}

En moyenne un individu produit à Lomé $0,45 \mathrm{~kg}$ de déchets par jour soit une production annuelle de 305340 tonnes dans le Grand Lomé. Plus de 89428 tonnes de déchets sont collectés et enfouis soit un taux de collecte de $29,29 \%$. Les déchets collectés sont constitués de putrescibles, de papier-cartons, de textiles, de verres, de métaux, de plastiques, de spéciaux, de fines (En majorité de sable), de combustibles non classés, des incombustibles non classés (Koledzi et al., 2011 ; Bodjona et al., 2012). On note 9000 tonnes de déchets valorisés en compost par des associations et/ou ONG.

Trois principaux acteurs interviennent dans la gestion des déchets dans le Grand Lomé à savoir :

$\checkmark \quad$ le secteur public représenté par les autorités locales (commune, préfecture,...);

$\checkmark \quad$ le secteur privé formé de petites et de grandes entreprises qui relient les activités des ONG et des autorités;

$\checkmark \quad$ le secteur informel composé soit par des particuliers rassemblés en association de quartier, soit par des petites entreprises non officielles, s'occupant soit de la précollecte en porte à porte, soit des matières recyclables.

\section{Secteur public et privé de la gestion des déchets dans le Grand Lomé}

L'organisation de la gestion des déchets à Lomé relève de la prérogative de la mairie de Lomé (Schéma). Elle gère toute la filière dans un cadre de Partenariat Public/Privé. Les Comités de Développement de Quartiers (CDQ), les structures de précollecte des ordures ménagères et certaines ONG axées sur les problématiques environnementales sont des partenaires privilégiés sur lesquels la mairie s'appuie pour une meilleure gestion des déchets dans la ville. Le système de collecte des déchets dans la ville de Lomé comporte trois phases principales que sont la pré-collecte (ou primaire) réalisée par les associations de quartiers, la post-collecte (ou secondaire) organisée par les entreprises sous le contrôle de la mairie à partir des centres de transit et la mise en décharge finale à Agoè Nyivé qui est gérée par l'entreprise ICON Sarl. Depuis 2018, la décharge d'Agoè Nyivé est fermée pour cause de saturation et avec l'opérationnalisation $\mathrm{du}$ nouveau centre d'enfouissement technique d'Aképé à Lomé.

A Lomé, la pré-collecte des déchets constitue la première phase dans la gestion des déchets. En effet, lors de la pré-collecte, les déchets sont pris en charge depuis leur origine, c'est-à-dire les ménages, jusqu'à leur dépôt dans les centres de transit où les entreprises de collecte prennent le relais. La pré-collecte par apport volontaire, consiste à l'acheminement des déchets par la population elle-même, à l'aide de sacs en plastique, des seaux de peinture de récupération, d'une brouette ou autre contenant vers un centre de transit situé à une distance maximale de 250 $\mathrm{m}$ des habitations.

La pré-collecte, organisée par les associations de quartier, consiste à ramasser les déchets chez les particuliers en porte à porte. Ces associations de pré-collecte, déclarées ou non, sont plus d'une centaine ce qui permet de par leur nombre de couvrir la totalité des quartiers de la ville.

Cette activité est effectuée le plus souvent par des jeunes gens sans emploi.

La collecte est assurée par les entreprises qui ont signé un contrat avec la Direction Des Services Techniques (DST) de la mairie. La collète secondaire, effectué par les camions et les bennes tasseuses des ménages vers la décharge finale à Agoè. $\mathrm{Ce}$ maillon est confié aux grosses et moyennes entreprises disposant des engins adaptés comme les bennes basculantes et tasseuses, les 
tracteurs-remorques. Ces entreprises sont payées par mois sur le tonnage collecté.

L'opération de la collecte est au cœur du processus de la gestion des déchets. C'est une opération d'ordre public qui rentre dans le cadre de la protection de la santé des populations ainsi que pour assurer une meilleure qualité de vie. Elle consiste en le ramassage et le regroupement des déchets en vue de leur transport. Ce dernier maillon est effectué par les grosses entreprises avec des engins comme les bennes basculantes, les bennes tasseuses, les chargeurs, les bulldozers et les compacteurs. Ce mode de gestion des déchets à Lomé au Togo est semblable à ceux de certains pays en développement (Hysacam 2008 ; Topanou 2012).

\section{Identification des travailleurs du secteur informel de la gestion des déchets}

Cette activité est fréquemment insalubre, souvent risquée, parfois même prohibée selon les lieux. En outre, le regard porté par la société sur les personnes qui procèdent à la récupération informelle des déchets est souvent dépréciatif : à l'exception de quelques cas de personnes (Environ 10\%) le faisant dans un but scientifique ou artistique ou de bricolage. Elle est, généralement, pratiquée que par ceux qui n'ont guère d'autres choix, par exemple des habitants des bidonvilles des pays en développement. Elle fait partie des activités de l'économie informelle.

Les travailleurs du secteur informel des déchets à Lomé sont constitués des pré- collecteurs, des récupérateurs de décharges et dépotoirs (30\% des personnes enquêtées), les récupérateurs ambulants (10\% des personnes enquêtées), les acheteurs ambulants (55\% des personnes enquêtées) et les grossistes (5\% des personnes enquêtées).

Un pré-collecteur informel est un individu ou groupe d'individus regroupé en association ou en ONG et faisant la collecte des déchets de porte en porte. Ils signent directement des contrats avec des ménages. Les déchets collectés sont convoyés dans les points de transit aménagés par la mairie.

Les récupérateurs de décharge ou de dépotoirs sont des individus cherchant des matériaux recyclables directement dans la décharge finale ou dépotoirs intermédiaires. Ces matériaux sont principalement des métaux, du plastique dur et du verre (Figure $3)$.

Les récupérateurs ambulants sont des individus qui circulent dans les quartiers afin d'acheter des déchets recyclables.

Les récupérateurs intermédiaires ou grossistes jouent un rôle de revendeurs des matériaux récupérés pour les industries, population et les exportateurs. Ils broient ces matériaux ou les compressent avant de les revendre aux industriels ou aux exportateurs.

L'intégration des acteurs informels dans la filière de traitement des déchets a un impact positif sur l'environnement, l'efficience de la filière et les revenus des populations défavorisées.

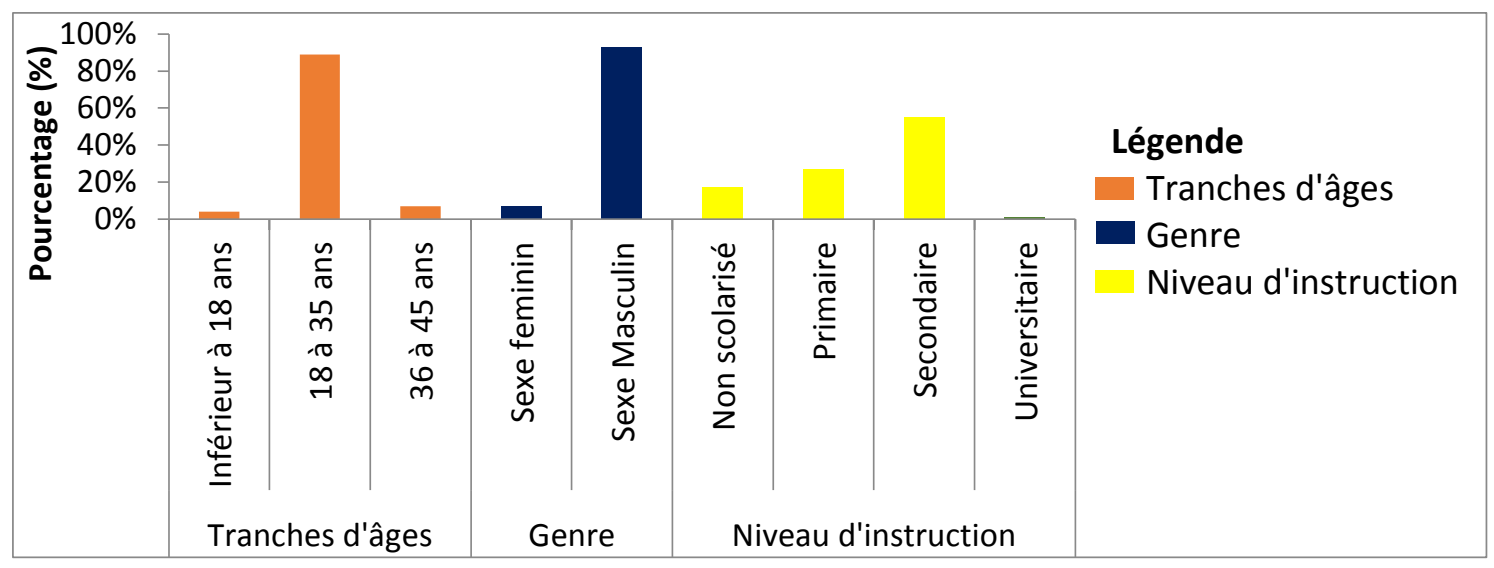

Figure 2: Répartition des tranches d'âge, le genre et le niveau d'instruction des personnes enquêtées. 


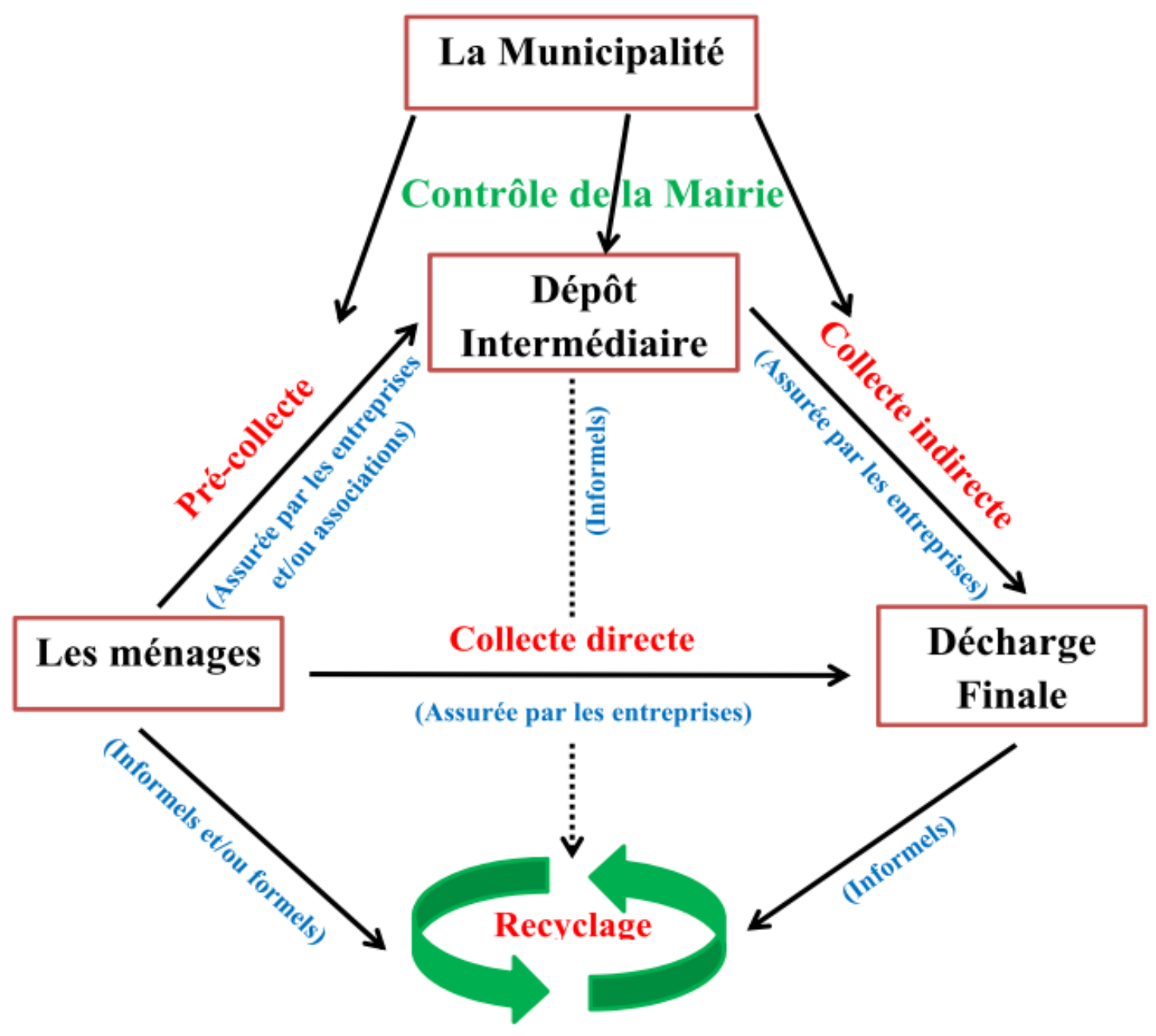

Schéma: Organisation de la gestion des déchets dans le Grand Lomé.

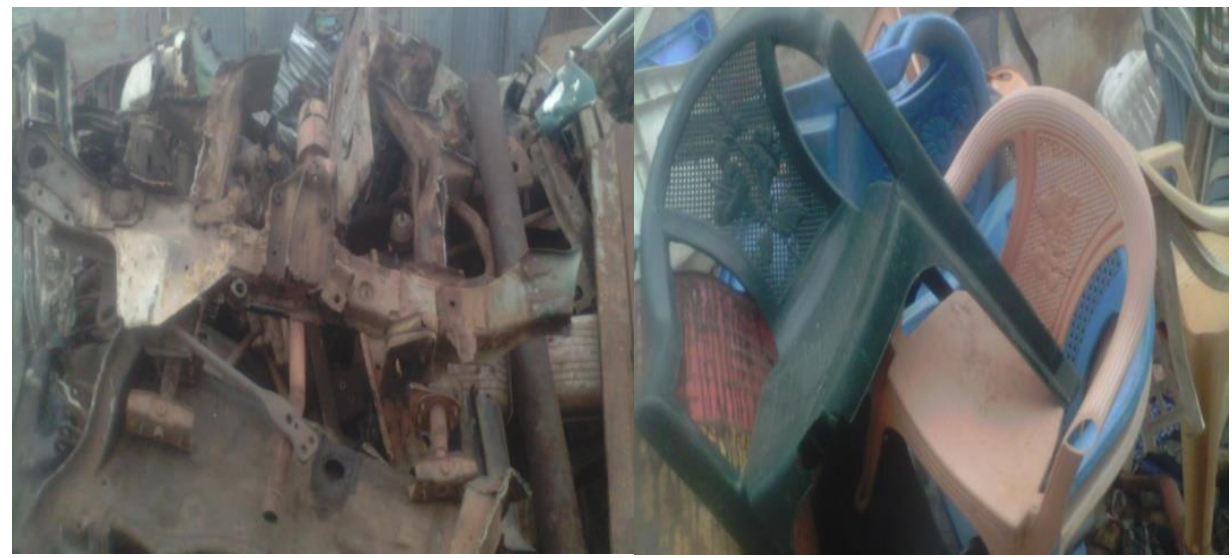

Figure 3: Déchets recyclables collectés par les informels. 


\section{DISCUSSION}

\section{Etat de la gestion des déchets dans le Grand Lomé}

La gestion des déchets dans le Grand Lomé est confrontée à des contraintes qui entravent son développement. Ces contraintes sont institutionnelles, organisationnelles, techniques, financières et comportementales. Parmi lesquelles on peut citer :

$\checkmark \quad$ la contrainte majeure est financière : les ressources financières prévisibles ne sont pas à la hauteur des besoins présents ;

$\checkmark \quad$ la négligence de l'Etat de faire appliquer les lois, modestes soient-elles ;

$\checkmark$ une multitude d'intervenants dans le secteur de la gestion des déchets avec des chevauchements d'attributions et une absence totale de coordination ;

$\checkmark \quad$ l'état de dégradation des infrastructures;

$\checkmark$ des institutions du secteur affaiblies, avec un personnel démotivé, incompétent, dont le savoir-faire s'est érodé ;

$\checkmark$ absence de sensibilisation sur l'assainissement et ses avantages pour la santé en multipliant des communications pour le changement de comportement.

Ces contraintes seront probablement dues au fait qu'à Lomé, la gestion des déchets n'a pas toujours été une préoccupation comme pour les autres villes des pays en développement (Koledzi et al., 2014). Aussi, aujourd'hui malgré plusieurs projets dans le domaine de la gestion des déchets qu'a piloté la municipalité, on constate toujours une multitude de dépotoirs aux abords des rues avec pour exutoire le remblaiement d'une carrière (Koledzi et al., 2014). Ce phénomène de multitude de dépotoirs sauvages qui n'est pas sans conséquences sur le bien-être de l'homme et l'environnement, peut être observé dans la plupart des villes africaines (Thonart et al., 2005 ; Ahoussi et al., 2008; Parrot et al., 2009). Une des raisons qui peut expliquer le disfonctionnement de la gestion des déchets à Lomé, est la non prise en compte par les autorités compétentes des résultats de recherche sur la gestion des déchets (Bodjona, 2012; Segbeaya, 2012; Koledzi, 2011).

\section{Caractéristiques sociodémographiques des personnes enquêtées}

Les enquêtes de terrain révèlent que la majorité des personnes enquêtées a un âge compris entre 18 et 35 ans et sont de sexe masculin. Ces genres d'activités exigent beaucoup d'efforts physiques, ce qui justifierait la présence exclusive des jeunes gens.

Les résultats de l'enquête montrent que la majorité des gens qui s'adonnent à ce métier sont des personnes de sexe masculin environ 93\%. Les femmes interrogées, exercent ce métier de manière occasionnelle. La prédominance des hommes dans ce secteur d'activité serait due à la pénibilité de l'activité qui exige des efforts physiques énormes, de nombreuses difficultés (longue distance à parcourir, la familiarisation quotidienne avec les odeurs émanant des ordures, etc.) et aux équipements rustiques utilisés pour la précollecte et la recherche des matériaux de récupération. Ces personnes en quête d'emploi sont pour la plupart déscolarisés avec un niveau d'instruction assez faible d'où les difficultés d'organisation constatées dans le secteur. Les statistiques du niveau d'instruction des travailleurs informels de déchets illustrent bien cet état de fait indiquant $17 \%$ non scolarisés, $27 \%$ niveau primaire, $55 \%$ niveau secondaire et $1 \%$ niveau universitaire. Par contre, Krou (2017) dans ses travaux a trouvé que au centre du Togo pour le quartier Tchawanda, $50 \%$ des répondants ont un niveau d'étude secondaire, $33 \%$ ont un niveau primaire, $13 \%$ ont un niveau supérieur ou universitaire et $4 \%$ des enquêtés sont analphabètes et pour le quartier Kpangalam, on relève $2 \%$ d'analphabètes, $40 \%$ ayant un niveau primaire, $43 \%$ un niveau secondaire et $15 \%$ le niveau universitaire. 


\section{Structuration du secteur informel de la gestion des déchets}

Ce secteur est très faiblement structuré puisque la plus grande partie des personnes travaille sans aucune autorisation. Le fait d'être informels, implique régulièrement une assimilation avec des activités criminelles ou illégales, et représente un obstacle certain aux tentatives d'inclusion (Bodjona et al., 2014). Ce manque de structuration ne permet pas d'avoir accès aux appuis financiers diminuant ainsi leur efficacité. Une organisation de ce secteur permettra de rendre plus performant leur activité pour une meilleur gestion des déchets dans le Grand Lomé.

Le secteur informel a développé une large expérience dans la récupération et le recyclage de matériaux valorisables. $\mathrm{La}$ récupération de matériaux a des avantages financiers et environnementaux (Bodjona et al., 2014).

Les activités du secteur informel de récupération des déchets solides ménagers dans le Grand Lomé, fournissent de l'emploi pour un nombre important des citoyens. Ce secteur reste le premier générateur d'emploi en matière de recyclage. Le secteur informel de la gestion des déchets contribue à la diminution de l'empreinte carbone et les émissions de substances polluantes issues de l'extraction de ressources naturelles pour la fabrication de nouveaux produits (Scheinberg et al., 2010; Gunsilius et al., 2011). En revanche, les activités du secteur informel se caractérisent par des conditions de travail déplaisantes et dangereuses. Les individus de ce secteur sont rarement protégés contre les maladies et les blessures liées au travail. Le travail $\mathrm{du}$ secteur informel demeure généralement précaire et exécuté dans des conditions sanitaires et de sécurité très mauvaises, parfois inhumaines. Les récupérateurs courent de graves risques en se blessant, surtout dans les dépotoirs et à la décharge finale où ils peuvent être écrasés par des camions ou victimes de glissements et d'éboulements de terrain et d'incendies pourtant, ils contribuent énormément à la gestion efficace des déchets (Gunsilius et al., 2011). Ils sont aussi exposés à de grandes quantités de gaz toxiques sur la décharge. Les risques sanitaires résultent de l'exposition à des substances toxiques et des blessures lors de la recherche des matériaux recyclables ou lors de la casse de ces matériaux après l'achat en vue de la réduction de leurs volumes.

Malgré les considérations régressives des récupérateurs informels des déchets recyclables à Lomé, ils constituent un maillon important dans la chaine de la gestion des déchets. Ils contribuent à un tant soit peu à la réduction du volume des déchets à enfouir à travers la récupération du matériel recyclable puisque la gestion des déchets, quel que soit le pays, a pour but final la réduction du volume des déchets destinés à la décharge finale (Aloueimine et al., 2006; Mosler et al., 2006).

\section{Conclusion}

L'état des lieux effectué sur la gestion des déchets dans le Grand Lomé a permis d'identifier trois principaux acteurs en charge de la gestion des déchets qui sont le secteur public, le secteur privé et le secteur informel. L'organisation de la gestion des déchets à Lomé relève de la prérogative de la mairie de Lomé. Le secteur informel joue un rôle non négligeable dans la chaine de la gestion des déchets dans le Grand Lomé. Malheureusement, il n'existe aucun mécanisme de suivi et d'accompagnement de ce secteur à Lomé. Une organisation du secteur informel de manière juste et efficace permettrait de renforcer d'avantage ce maillon de la gestion de déchets. Les initiatives de gestion inclusive permettront de faire évoluer la situation des travailleurs informels. Une vraie restructuration du secteur des déchets avec la mise en place d'un schéma directeur d'assainissement dans la ville de Lomé permettra de rendre efficace ce secteur. 
Comme recommandation, il faut une protection et insertion sociale des travailleurs informels des déchets sur les aspects du droit, les réglementations $\mathrm{du}$ travail, la formalisation, la santé et la sécurité au travail.

\section{CONFLIT D'INTERETS}

Les auteurs déclarent qu'il n'y a aucun conflit d'intérêts pour ce travail.

\section{CONTRIBUTIONS DES AUTEURS}

MBB est l'instructeur des travaux. Il a guidé EK dans son travail. Il a contribution à l'analyse des résultats. EK a travaillé sur le terrain pour la collecte des données et a rédigé ce projet d'article.

\section{REMERCIEMENTS}

Toutes nos reconnaissances au Directeur du Laboratoire GTVD de la Faculté des Sciences de L'Université de Lomé, le Professeur Gado TCHANGBEDJI, pour nous avoir autorisés d'effectuer nos travaux de recherche dans son Laboratoire.

\section{REFERENCES}

Ahoussi KE, Soro NG, Soro B, Lasm T, Oga MS, Zade SP. 2008. Ground water pollution in africa biggest towns: case of the town of Abidjan (CI). Environnemental Journal Scientific Research, 2:302-316.

Aloueimine S, Matejka G, Zurbrugg C, Sidi Mohamed ME. 2006. Caractérisation des Ordures ménagères à Nouakchott: Partie II: Résultats en Saison Sèche et en Saison Humide. Déchets-Revue Francophone d'Ecologie Industrielle, $44: 8 \mathrm{p}$.

Aloueimine SO. 2006. Méthodologie de caractérisation des déchets ménagers à Nouakchott (Mauritanie) : Contribution à la gestion des déchets et outils d'aide à la décision. Thèse de doctorat, Université de Limoges, Limoges.

Bodjona BM, Bromblet H, Kolani L, Pierrat A. 2014. Prise en compte des travailleurs du secteur informel des déchets en Afrique et dans les caraïbes. Présentation synthétique de l'état des lieux et du retour d'expérience. Plateforme RéSources. www.plateforme-resources.org.

Bodjona BM. 2012. Contamination du sol de la décharge finale de la ville de Lomé par les métaux lourds : Cas du plomb, cadmium, nickel, cuivre et du zinc. Thèse de doctorat $\mathrm{N}^{\circ}$ 455/FDS, Université de Lomé, Togo.

Bodjona BM, Kili AK, Tchegueni S, Kennou B, Tchangbedji G, El Meray M. 2012. Evaluation de la quantité des métaux lourds dans la décharge d'Agoè (LoméTogo) : cas du plomb, cadmium, cuivre, nickel et zinc. Int. J. Biol. Chem. Sci., 6(3): $\quad 1368-1380 . \quad$ DOI: http://dx.doi.org/10.4314/ijbcs.v6i3.38.

Gunsilius E, Spies S, García-Cortés S, Medina M, Dias S, Scheinberg A, Sabry W, Abdel-Hardy N, Florisela dos Santos A, Ruiz S. 2011. Recovering resources, creating opportunities integrating the informal sector into solid waste management. Eschborn, Deutsche Gesellschaft für International Zusammenarbeit (GIZ), 48 p.

Harpet C. 2003. De l'anthropologie des décharges à l'évaluation interdisciplinaire des risques sanitaires. Natures, Sciences, Sociétés, 11(3): 361370. DOI:10.1016/S12401307(03)00116-X.

Hysacam 2008. Clean Mecanism Development Project Disign Form (CDM-PDD) Version 3. Apex Conseil, $62 \mathrm{p}$.

Koledzi KE, Agbebavi J, Baba G, Koffi D, Matejka G. 2014. Gestion des déchets dans les villes en développement : transfert, adaptation de schéma et sources de financement. Revue Déchets Sciences et Techniques - $\mathrm{N}^{\circ} 68$.

Koledzi KE, 2011. Valorisation des déchets solides urbains dans les quartiers de 
Lomé (Togo): approche méthodologique pour une production durable de compost. Thèse de doctorat $\mathrm{N}^{\circ}$ 04-2011, Université de Limoges, Limoges.

Koledzi KE, Baba G, Feuillade G, Matejka G. 2011. Caractérisation physique des déchets solides urbains à Lomé au Togo, dans la perspective du compostage décentralisé dans les quartiers. Revue Déchets Sciences et Techniques - N ${ }^{\circ} 59$.

Krou NM. 2017. Evaluation de la quantité et de la qualité de la matière organique contenue dans les ordures ménagères de la ville de Sokodé: cas des quartiers Kpangalam et Tchawanda. Master recherche $\mathrm{N}^{\circ} 111$ / FDS, Université de Lomé, Togo.

Mosler HJ, Drescher S, Zurbrügg C, Rodriguez TC, Miranda OG. 2006. Formulating waste management strategies based on waste management practices of households in Santiago de Cuba, Cuba. Habitat International, 30(4): 849-862.

Moreno-Sánchez RdP, Maldonado JH. 2006. Surviving from garbage: The role of informal waste-pickers in a dynamic model of solid-waste management in developing countries. Environment and Development Economics, 11(3): 371391.

Parrot L, Sotamenou J, Kamgnia DB. 2009. Municipal solid waste management in Africa: Strategies and livelihoods in
Yaoundé, Cameroon. Waste Management, 29(2): $\quad$ 986-995. DOI:10.1016/j.wasman.2008.05.005

Scheinberg A, Simpson M, Gupt Y. 2010. The Economics of the Informal Sector in Solid Waste Management. Eschborn, Allemagne, CWG - Collaborative Working Group on Solid Waste Management in Low- and Middleincome Countries GIZ - Deutsche Gesellschaft für Internationale Zusammenarbeit (GIZ) GmbH, 34 p.

Segbeaya KN, 2012. Évaluation de l'impact des déchets ménagers de la ville de Kara sur la qualité de la rivière Kara (Togo). Thèse de doctorat $\mathrm{N}^{\circ} 399$ / FDS, Université de Lomé, Togo.

Thonart P, Sory D. 2005. Guide pratique sur la gestion des déchets ménagers et des sites d'enfouissement techniques dans les pays $d u$ sud. Collection points de repèreOIF.

Topanou KAN. 2012. Gestion des déchets solides ménagers dans la ville d'Abomey- Calavi (Bénin): Caractérisation et essais de valorisation par compostage. Thèse de Doctorat $\mathrm{N}^{\circ}$ 25-2012/FDCA/FAST/UAC.

Wilson DC, Velis C, Cheeseman C. 2006. Role of informal sector recycling in waste management in developing countries. Habitat International, 30(4): 797-808. 